\title{
A note on the Relationship between Spatial Recognition and Behavior while Walking in the City
}

\author{
Kaori Ito $^{\text {a, }}{ }^{\text {, Yusuke Sakurai }}{ }^{\mathrm{b}}$, Yuri Fujita ${ }^{\mathrm{c}}$, Andrew Burgess ${ }^{\mathrm{d}}$ \\ ${ }^{a}$ Department of Architecture, Faculty of Science and Technology, Tokyo University of Science, kaori @ rs.noda.tus.ac.jp \\ ${ }^{b}$ Taisho Pharmaceutical Holdings \\ ${ }^{c}$ Dentsu \\ ${ }^{d}$ Freelance \\ * Corresponding author
}

Keywords: Spatial recognition, Smartphone, Map application, Sense of direction

\begin{abstract}
:
The experience of walking in the city has changed since the diffusion of smartphones with communication and GNSSbased navigation technologies. Accordingly, user's recognition of geographical space has also been changed; McCullough and Collins (2018) suggest that less spatial knowledge is gained while using GNSS-equipped devices while way-finding than by using paper maps or no tools at all; Ishikawa et al. (2008) suggest that GNSS-based navigation device users make larger direction errors and produce sketch maps with poorer topological accuracy after way-finding.
\end{abstract}

This study focuses on information gathering, decision-making and recognition of geographical space while 'strolling' in the city with a smartphone. This abstract describes the outline of the experiment and early stages of the analysis, especially relationships between spatial recognition through sketch maps and behavior while walking.

We organized an experiment in which nineteen undergraduate and graduate students (ten men and nine women) were asked to walk by him/herself from a given starting point to a final destination. The participants were told that they did not need to follow the direct route to the final destination and could visit any places in the neighborhood as long as they travelled on foot and arrived at the final destination before the sunset. All participants were smartphone users. Each participant walked with his/her own smartphone and used it as usual. Additionally, they carried GPS loggers with them. They were told to take screenshots when they used their smartphones in order to record what application they used and how they used it. The study area was Omotesando/Harajuku, a popular shopping neighborhood in Tokyo, Japan. The starting point was Omotesando subway station and the final destination was Kitasando subway station. The straightline distance between two stations is approximately $1.6 \mathrm{~km}$, and the shortest route is approximately $2.0 \mathrm{~km}$. Additionally, participants were required to answer a questionnaire before the experiment related to their daily smartphone usage and their sense of direction, based on Takeuchi (1992)'s Sense of Direction Questionnaire-Short Form (SDQ-S), and then draw sketch maps of their travel routes and be interviewed about their decision-making following the experiment.

The average travel time was 124 minutes. The average number of places they visited was 5.4. All participants used map applications on their smartphones while walking. The average number of map application uses was 5.5. There was considerable variation in their routes.

We classified their sketch maps into three groups: route only (group 1), map along route (group 2), and route on map (group 3). There were ten sketch maps in group 1, six in group 2 and three in group 3. A typical sketch map in each group is shown in Figure 1.
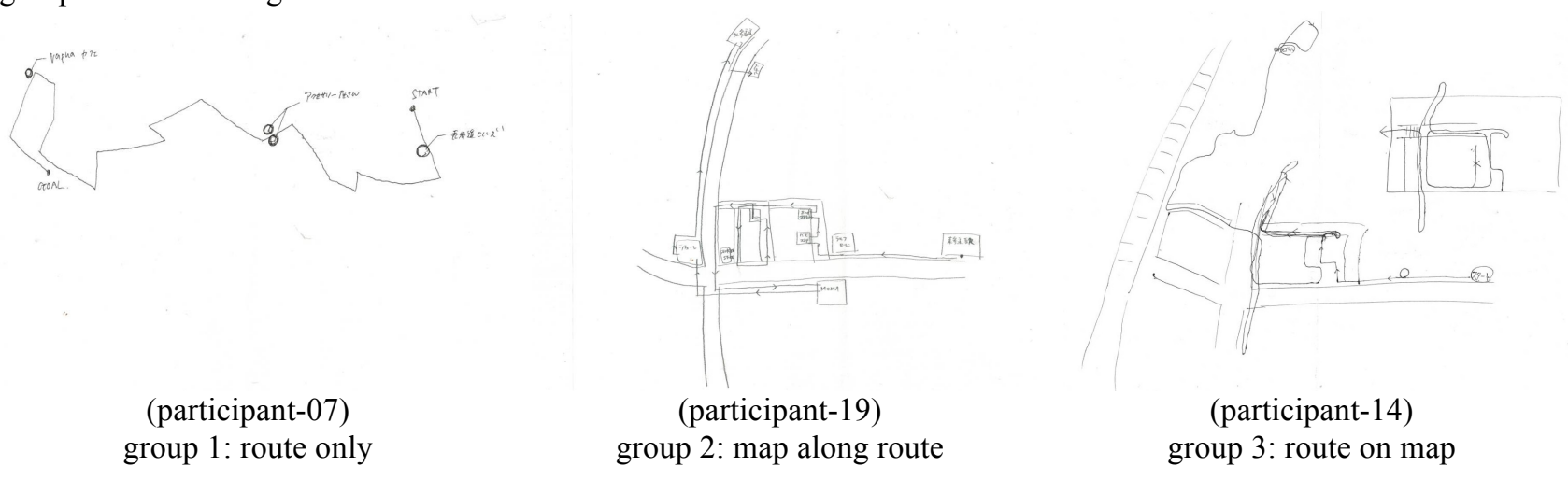

Figure 1. Sketch map samples from three groups 
T-tests found that the participants who drew group 1 or group 2-type sketch maps used map applications significantly more times than those who drew group 3-type sketch maps and that the participants who drew group 1-type sketch maps have a significantly lower sense of direction than those who drew group 2 or group 3-type sketch maps. Additional t-tests found that those who drew group 3-type sketch maps visited significantly more places than those in group 2 and group 1 and that those in group 2 visited significantly more places than those in group 1 . This also holds for travel times.

Although causal relationships are not clear, the results imply that those who have a lower sense of direction and use smartphone map applications more frequently while walking tend to recognize the physical environment locally along their routes without geographical context. Additionally, those who travelled longer times and visited more places tend to locate their routes in geographical context.

Further investigation of the experiment data is required to better understand the relationships among information gathering, decision-making and recognition of geographical space while walking in the city.

\section{Acknowledgements:}

The study was supported by JSPS KAKENHI Grant Number JP17H00839.

\section{References:}

David McCullough and Rebecca Collins (2018), “Are we losing our way?" Navigational aids, socio-sensory wayfinding and the spatial awareness of young adults, Area. 2018;00:1-10 https://doi.org/10.1111/area.12478

Toru Ishikawa, Hiromichi Fujiwara, Osamu Imai, Atsuyuki Okabe (2008), Wayfinding with a GPS-based mobile navigation system: A comparison with maps and direct experience, Journal of Environmental Psychology 28(1), 74-82.

Yoshiaki Takeuchi (1992), Sense of Direction and Its Relationship with Geographical Orientation, Personality Traits and Mental Ability, Japanese Journal of Educational Psychology 40, 47-53. (Japanese) 